\title{
A Case of Wound Dual Infection with Pasteurella Dagmatis and PAsteurella CANis Resulting From a Dog Bite - Limitations of ViteK-2 System IN EXACT IDENTIFiCATION OF PASTEURELLA SPECIES
}

\author{
T. Akahane ${ }^{1,4}$, M. Nagata ${ }^{4,6}$, T. Matsumoto ${ }^{3}$, N. Murayama ${ }^{1}$, A. Isaka ${ }^{2}$, T. Kameda ${ }^{2}$, M. Fujita ${ }^{2}$, K. Oana ${ }^{4,5}$, \\ Y. Kawakami 4,5 \\ Division of Clinical Microbiology, ${ }^{1}$ Department of Clinical Laboratories, ${ }^{2}$ Department of Emergency and Critical Care, \\ Azumino Red Cross Hospital, Azumino, Japan, \\ ${ }^{3}$ Department of Laboratory Medicine, Shinshu University Hospital, Matsumoto, Japan, \\ ${ }^{4}$ Division of Infection Control and Microbiological Regulation, Department of Health and Medical Sciences, \\ Shinshu University Graduate School of Medicine, \\ ${ }^{5}$ Division of Clinical Microbiology, Department of Biomedical Laboratory Sciences, School of Health Sciences, Shinshu University \\ School of Medicine, Matsumoto, Japan, \\ ${ }^{6}$ Division of Clinical Microbiology, Department of Clinical Laboratories, Keio University Hospital, Tokyo, Japan
}

\begin{abstract}
Background: Pasteurella species, widely known as indigenous organisms in the oral and gastrointestinal floras of many wild and domestic animals, are important pathogens in both animals and humans. Human infections due to Pasteurella species are in most cases associated with infected injuries following animal bites. We encountered a rare case of dual infections caused by different two Pasteurella species occurred in a previously healthy 25-year-old female sustaining injury by a dog-bite.

Methodology: Exudates from the open wound of her dog-bite site, together with the saliva of the dog were submitted for bacteriological examination. Predominantly appearing grayish-white smooth colonies with almost the same colonial properties but slightly different glistening grown on chocolate and sheep blood agar plates were characterized morphologically by Gram's stain, biochemically by automated instrument using Vitek 2 system using GN cards together with commercially available kit system, ID-Test HN-20 rapid panels, and genetically by sequencing the $16 \mathrm{~S}$ rRNA genes of the organism using a Taq DyeDeoxy Terminator Cycle Sequencing and a model 3100 DNA sequencer instrument.

Results: The causative isolates from the dog-bite site were finally identified as $P$. canis and $P$. dagmatis from the findings of the morphological, cultural, and biochemical properties together with the comparative sequences of the 16S rRNA genes. Both the isolates were highly susceptible to many antibiotics and the patient was successfully treated with the administration of so-called the first generation cephalosporin, cefazolin followed by so-called the third generation cephalosporin, cefcapene pivoxil. The isolate from the dog was subsequently identified as $P$. canis, the same species as the isolate from the patient.
\end{abstract}

Conclusions: To the best of our knowledge, this was the second report of a dual infection with Pasteurella species consisting of $P$. dagmatis and $P$. canis resulting from a dog-bite, followed by the first report of dual infections due to $P$. dagmatis and P. multocida in 1988. Our isolate finally identified as $P$. dagmatis was misidentified as $P$. pneumotripica by means of the Vitek 2 system. The species name "P. dagmatis" was not included in the database of the system. It is also important for routine clinical microbiology laboratories to know the limitation of the automated Vitek 2 system for the accurate identification of Pasteurella species especially $P$. dagmatis. It should be emphasized that there still exists much room for improvement in Vitek 2 system. Significant improvement of Vitek 2 system especially in the identification of Pasteurella species is urgently desired.

Key words: dual wound infection, dog bite, Vitek 2 system, misidentification, Pasteurella dagmatis, Pasteurella canis, Pasteurella pneumotropica

\section{INTRODUCTION}

Pasteurella species are small, nonmotile, gram-negative, bipolar-staining facultative anaerobes present in the oropharynx of the majority of healthy dogs and cats, and are the causative agents of zoonotic infections in humans [1-7]. The frequent occurrences of infections due to Pasteurella species have been documented to date accompanied by the recent popularity of pets. Indeed, human pasteurellosis are most often caused by dog and cat bites, resulting in cellulitis and subcutaneous abscesses [8-10]. Pasteurella species are infrequently caused systemic infectious diseases and mostly strike in patients with underlying diseases. $P$. multocida is the most recurrent species in human in- 
fections [11], but other species may be involved, such as $P$. canis, and $P$. dagmatis $[7,12]$. Automated systems are generally used for the identification of Pasteurella isolates. However, the failure of commercial systems to satisfactorily identify microorganisms is of concern, and unusual identification should be correlated with patient's clinical pictures. We are reporting here a rare case of dual infections due both to $P$. canis and to $P$. dagmatis, focusing on the limitations of automated Vitek 2 system using GN cards (Nippon sysmex bioMérieux, Co., Ltd., Tokyo, Japan) as well as commercially available kit system, ID-Test HN20 rapid panels (Nissui Pharmaceutical Co., Ltd., Tokyo, Japan), for exact identification of Pasteurella species.

\section{CASE RePort}

A previously healthy 25-year-old female patient was admitted to the department of emergency and critical care in Azumino Red Cross Hospital, Azumino, 3998292, Japan on March 3 in 2010. She complained of a severe inflammation accompanied by sensations of burning along the circumference of thumb root part in her left hand. She was bitten by her pet dog two days before, and her injured area was $15 \mathrm{~mm}$ in length and $10 \mathrm{~mm}$ in depth. An X-ray examination on her admission manifested that she had no fracture of the bones. Skin examination of her left hand revealed inflammation, swelling, and sharp pain without purulent discharges. No regional lymphadenopathy was noted. Two distinctive Pasteurella isolates were recovered as the causative agents. After treating the injured area with the gentamicin-ointment, she was initially administered for 3 days with cefazolin (1g) as intravenous drip infusion, and then switched to oral administration of cefcapene pivoxil $(300 \mathrm{mg} /$ day) therapy for additional 5 days. Her skin inflammation, swelling, and tenderness disappeared, but she felt a slight sensation at the injured site on 9 March in 2010, and she continued the oral administration of cefcapene pivoxil $(300 \mathrm{mg} /$ day) until her last consultation on 12 March in 2010, when she was confirmed the complete recovery.

\section{Cultural Findings of Each Medium.}

The exudates from the open wound of her dog-bite site submitted for bacteriological examination were cultivated at $35^{\circ} \mathrm{C}$ for 24 hours under an ambient air on Sheep Blood agar (Nippon Becton Dickinson Co., Ltd., Tokyo, Japan.), on Chocolate agar (Nippon Becton Dickinson Co., Ltd., Tokyo, Japan.), and on modified Drigalski agar (Nippon Becton Dickinson Co., Ltd., Tokyo, Japan) plates. Incubation in the anaerobic chamber at $35^{\circ} \mathrm{C}$ for 72 hours yielded no detectable strictly anaerobic microorganisms. Although no-visible or dim growth was observed on modified Drigalski agar (Nippon Becton Dickinson) both the Sheep Blood agar (Nippon Becton Dickinson) and Chocolate agar (Nippon Becton Dickinson) plates exhibited distinctly positive growth for numerous bacterial cells, representing almost the homologous but discriminative two kinds of non-pigmented, opaque, and small to tiny colonies with a diameter of about 1.5 to $2 \mathrm{~mm}$, designated as strain-A and strain-B, respectively. Colonies of the strain-A grown after overnight incubation at $35^{\circ} \mathrm{C}$ on the Sheep Blood agar (Nippon Becton Dickinson) plates in an ambient air demonstrated to be smooth and slightly glistening and reminiscent of Haemophilus or Aggregatibacter species. On the other hand, colonies of the strain- $\mathrm{B}$ were grayish white and smooth in shape and resembled Enterococcus species.

In addition, the oral swabs and the saliva juice specimens from her pet dog were also submitted to our laboratory for bacteriological examination and successfully yielded numerous colonies designated as strain- $C$ with almost exactly the same colonial types as those of strain-B from her injured site were cultivated on both the Sheep Blood agar (Nippon Becton Dickinson) and the Chocolate agar (Nippon Becton Dickinson) plates.

The isolates of strain-A, strain-B, and strain-C were characterized morphologically by Gram's stain, biochemically by automated instrument, Vitek 2 system using GN cards (Nippon sysmex bioMérieux) together with commercially available kit system, ID-Test HN20 rapid panels (Nissui Pharmaceutical), and genetically by sequencing the $16 \mathrm{~S}$ rRNA genes of the organism [13] using a Taq DyeDeoxy Terminator Cycle Sequencing and a model 3100 DNA sequencer instrument [14].

\section{Microbiological Properties of the Isolates.}

The causative agents of two isolates, strain-A and strain-B, from exudates of her injured area, with discriminative colonial morphology were subjected to microbiological examinations. Both the isolates displayed good growths on Sheep blood agar (Nippon Becton Dickinson) and on Chocolate agar (Nippon Becton Dickinson) plates, but exhibited faint and faded or novisible growth on modified Drigalski agar (Nippon Becton Dickinson) plates. They exhibited facultatively anaerobic Gram-negative coccobacilli to short rodshaped morphology, demonstrating positive catalase reactions with formation of oxygen gas bubbles after emulsifying a fresh colony in a drop of $5 \% \mathrm{H}_{2} \mathrm{O}_{2}$ on a slide-glass, and were also oxidase positive with the paper strip (Wako Pure Chemical Industry Co., Ltd., Tokyo, Japan) method. Biochemical characterizations of the isolates were carried out with the Vitek 2 system using GN cards (Nippon sysmex bioMérieux), together with ID-Test HN20 rapid (Nissui Pharmaceutical) kit (Table 1) panels. Inoculated cards and kit panels were kept at $35^{\circ} \mathrm{C}$ in the atmosphere, and final readings were carried out according to the instructions of the manufactures. As shown in Table 2, Vitek 2 GN cards (Nippon Sysmex bioMérieux) identified both the causative isolates as $91.3 \% \mathrm{P}$. pneumotropica for strain-A with good identification confidence level, and $99.0 \%$ P. canis for strain-B with excellent identification confidence level, after incubation for 8 and 7 hours, respectively. In addition, the isolate of strain-C from her pet dog was identified by the Vitek 2 GN cards (Nippon sysmex bioMérieux) as $99.0 \%$ P. canis with excellent identification confidence level, after incubation for 7 hours. 
Table 1. Differential biochemical characteristics of 3 Pasteurella isolates, Strain-A*, Strain-B*, and Strain-C*, obtained with IDTest HN20 Rapid panels.

\begin{tabular}{lccc}
\hline & $\begin{array}{c}\text { Strain-A* } \\
\text { Pasteurella dagmatis }\end{array}$ & $\begin{array}{c}\text { Strain-B* } \\
\text { Pasteurella canis }\end{array}$ & $\begin{array}{c}\text { Strain-C* } \\
\text { Pasteurella canis }\end{array}$ \\
\hline Acid from: & + & + & + \\
glucose & + & - & - \\
maltose & + & + & + \\
fructose & + & + & - \\
mannose & - & - & + \\
mannitol & + & + & - \\
trehalose & + & - & - \\
sucrose & - & - & + \\
lactose & + & + & + \\
xylose & + & + & + \\
Nitrate to nitrite & + & + & - \\
Catalase & + & - & + \\
Oxidase & + & + & - \\
Indole production & + & - & + \\
Urease activity & - & & + \\
Ornithine decarboxylase & - & + & + \\
ONPG\# reaction & & & + \\
\hline
\end{tabular}

*: See text in Microbiological Properties of the Isolates for the origins and the backgrounds of respective isolate. \#: ortho-nitrophenyl- $\beta$-D-galactopyranoside.

Table 2. Identification results obtained with Vitek 2 GN cards and ID-Test HN20 rapid panels compared with those by $16 \mathrm{~S}$ rRNA analyses.

\begin{tabular}{|c|c|c|c|}
\hline & 16S rRNA & Vitek 2 (GN card ) & ID-test(HN-20rapid ) \\
\hline Strain-A* & $\begin{array}{l}\text { Pasteurella dagmatis } \\
(559 / 559 \mathrm{bp} ; \\
\text { Homology } 100 \%)\end{array}$ & $\begin{array}{l}\text { Pasteurella pneumotropica } \\
\text { (Probability of } 91.3 \%, 8 \mathrm{~h} \text { ) } \\
\text { Good Identification }\end{array}$ & $\begin{array}{l}\text { Pasteurella dagmatis } \\
\text { Profile No. } 7517552 \\
100 \%\end{array}$ \\
\hline Strain-B* & $\begin{array}{l}\text { Pasteurella canis } \\
(540 / 541 \mathrm{bp} ; \\
\text { Homology } 99.8 \%)\end{array}$ & $\begin{array}{l}\text { Pasteurella canis } \\
\text { (Probability } 99.0 \%, 7 \mathrm{~h} \text { ) } \\
\text { Excellent Identification }\end{array}$ & $\begin{array}{l}\text { Pasteurella multocida } \\
\text { Profile No. } 7605152 \\
100 \%\end{array}$ \\
\hline Strain-C & $\begin{array}{l}\text { Pasteurella canis } \\
(409 / 409 \mathrm{bp} ; \\
\text { Homology 100\%) }\end{array}$ & $\begin{array}{l}\text { Pasteurella canis } \\
\text { (Probability } 99.0 \%, 7 \mathrm{~h} \text { ) } \\
\text { Excellent Identification }\end{array}$ & $\begin{array}{l}\text { Pasteurella multocida } \\
\text { Profile No. } 7615552 \\
100 \%\end{array}$ \\
\hline
\end{tabular}

*: See text in Microbiological Properties of the Isolates for the origins and the backgrounds of respective isolate.

However, as shown in Table 2, ID-Test HN20 rapid panels (Nissui Pharmaceutical) conduced to the different identification results; strain-A as $100 \% P$. dagmatis with the biochemical profile of 7517552 , strain-B as $100 \%$ P. multocida with the biochemical profile of 7605152 , and strain-C as $100 \%$ P. multocida with the biochemical profile of 7615552 , respectively. These discrepant identification results led us to approach the accurate identification of the isolates by the genetic examinations. Therefore, the $16 \mathrm{~S} \mathrm{rRNA}$ genes of the isolates were directly sequenced as described previously [7] using a Taq DyeDeoxy Terminator Cycle Sequencing kit (Applied Biosystems, Foster City, CA, USA) and a model 3100 DNA sequencer instrument (Applied Biosystems, Foster City, CA, USA). The sequences were retrieved from the Ribosomal Database
Project databases [14]. As clearly shown in Table 2, comparative sequence analyses disclosed strain-A with $100 \% 16 \mathrm{~S}$ rRNA sequence similarity to that of $P$. dagmatis, strain-B with $99.8 \%$ 16S rRNA sequence similarity to that of $P$. canis, and strain-C with $100 \% 16 \mathrm{~S}$ rRNA sequence similarity to that of $P$. canis, respectively. Based on the phenotypic and genetic properties, we finally identified the isolate as $P$. dagmatis for strain-A, as $P$. canis for strain- $\mathrm{B}$, and as $P$. canis for strain- $C$, respectively.

In addition, the minimum inhibitory concentrations (MICs) determined with the Vitek 2 AST-N025 panels (Nippon bioMérieux, Co., Ltd., Tokyo, Japan.) were shown in Table 3. Three isolates of strain-A, strain-B, and strain- $C$ were exceptionally highly susceptible to all of the antimicrobial agents provided by the cards. 
Table 3. Antimicrobial susceptibility of 3 Pasteurella Isolates against 17 agents provided by the Vitek 2 GN cards.

\begin{tabular}{|c|c|c|c|c|c|c|}
\hline \multirow[b]{2}{*}{ Antimicrobial agents } & \multicolumn{2}{|c|}{$\begin{array}{c}\text { Strain-A* } \\
\text { Pasteurella dagmatis }\end{array}$} & \multicolumn{2}{|c|}{$\begin{array}{c}\text { Strain-B* } \\
\text { Pasteurella canis }\end{array}$} & \multicolumn{2}{|c|}{$\begin{array}{c}\text { Strain-C* } \\
\text { Pasteurella canis }\end{array}$} \\
\hline & $\operatorname{MIC\# }(\mu \mathrm{g} / \mathrm{ml})$ & Category & $\operatorname{MIC} \#(\mu \mathrm{g} / \mathrm{ml})$ & Category & $\mathrm{MIC}(\# \mu \mathrm{g} / \mathrm{ml})$ & Category \\
\hline Ampicillin & $\leq 2$ & $\mathrm{~S}^{*}$ & $\leq 2$ & S & $\leq 2$ & $\mathrm{~S}$ \\
\hline Sulbactam $\cdot$ Ampicillin & $\leq 2$ & $\mathrm{~S}$ & $\leq 2$ & S & $\leq 2$ & $\mathrm{~S}$ \\
\hline Clavulanic Amoxicillin & $\leq 2$ & $\mathrm{~S}$ & $\leq 2$ & $\mathrm{~S}$ & $\leq 2$ & $\mathrm{~S}$ \\
\hline Piperacillin & $\leq 4$ & $\mathrm{~S}$ & $\leq 4$ & S & $\leq 4$ & $\mathrm{~S}$ \\
\hline Cefazolin & $\leq 4$ & $\mathrm{~S}$ & $\leq 4$ & S & $\leq 4$ & $\mathrm{~S}$ \\
\hline Cefotaxime & $\leq 1$ & $\mathrm{~S}$ & $\leq 1$ & S & $\leq 1$ & $\mathrm{~S}$ \\
\hline Ceftazidime & $\leq 1$ & $\mathrm{~S}$ & $\leq 1$ & $\mathrm{~S}$ & $\leq 1$ & $\mathrm{~S}$ \\
\hline Cefpime & $\leq 1$ & $\mathrm{~S}$ & $\leq 1$ & $\mathrm{~S}$ & $\leq 1$ & $\mathrm{~S}$ \\
\hline Imipenem & $\leq 1$ & $\mathrm{~S}$ & $\leq 1$ & $\mathrm{~S}$ & $\leq 1$ & $\mathrm{~S}$ \\
\hline Meropenem & $\leq 0.25$ & $\mathrm{~S}$ & $\leq 0.2$ & $\mathrm{~S}$ & $\leq 0.25$ & $\mathrm{~S}$ \\
\hline Aztreonam & $\leq 1$ & $\mathrm{~S}$ & $\leq 1$ & $\mathrm{~S}$ & $\leq 1$ & $\mathrm{~S}$ \\
\hline Gentamicin & $\leq 1$ & $\mathrm{~S}$ & $\leq 1$ & $\mathrm{~S}$ & $\leq 1$ & $\mathrm{~S}$ \\
\hline Amikacin & 4 & $\mathrm{~S}$ & $\leq 2$ & $\mathrm{~S}$ & $\leq 2$ & $\mathrm{~S}$ \\
\hline Minocycline & $\leq 1$ & $\mathrm{~S}$ & $\leq 1$ & $\mathrm{~S}$ & $\leq 1$ & $\mathrm{~S}$ \\
\hline Ciprofloxacin & $\leq 0.25$ & $\mathrm{~S}$ & $\leq 0.25$ & $\mathrm{~S}$ & $\leq 0.25$ & $\mathrm{~S}$ \\
\hline Levofloxacin & $\leq 0.25$ & $\mathrm{~S}$ & $\leq 0.251$ & S & $\leq 0.12$ & $\mathrm{~S}$ \\
\hline Sulfamethoxazole $\cdot$ Trimethoprim & $\leq 20$ & $\mathrm{~S}$ & 20 & S & $\leq 20$ & S \\
\hline
\end{tabular}

*: See text in Microbiological Properties of the Isolates for the origins and the backgrounds of respective isolate. \#: Minimum inhibitory concentration $※$ : Susceptible

\section{Discussion}

Pasteurella species, causative agents of zoonotic infections in humans, are the inhabitants in the oropharynx of the majority of healthy dogs and cats [1-7]. Indeed, they have been isolated from 20 to $30 \%$ of dog-bite wounds and more than $50 \%$ of cat-bite wounds [15]. Most Pasteurella infections occur in people who have frequent contact with pet animals [7]. Among the Pasteurella species, recently described species of the organism, called $P$. dagmatis, has previously been known as Pasteurella "gas", Pasteurella new species 1 or Pasteurella pneumotropica type Henriksen that is rarely implicated in human pathology $[7,16]$. However, P. dagmatis is often isolated simultaneously with other bacteria [17], and misidentification may have contributed to the slightly underestimated frequency of its isolation [18]. Pasteurella acquired from pets may cause a variety of infections, including tonsillitis, sinusitis, and epiglottiditis $[19,20]$. We report a rare case of $P$. dagmatis infection together with $P$. canis resulting from a dog bite.

To the best of our knowledge, this is the sixth human case report of $P$. dagmatis isolation. The incidence of $P$. dagmatis infection has been increasing in many countries. The previously described five cases were as follows; one case was $P$. dagmatis endocarditis, occurred in a healthy man after a cat-bite [21], the second case was complicated by vertebral osteomyelitis, involved the native mitral valve of a cirrhotic woman with a known history of animal contact [22], the third case was spondylodiscitis in a diabetic patient [23], the fourth case was a septicemia due to $P$. dagmatis in a diabetic patient [12], and the fifth case was a wound infection together with $P$. multocida resulting from a cat bite [24]. The above five cases demonstrated that continuous contact with small animals such as dogs or cats might be a risk factor for transmission of Pasteurella species for humans. Our sixth case was also apparently associated with a dog bite.

As far as we can predict, the dual or simultaneous infectious diseases due to two different Pasteurella species have been documented only once in 1988, just descrived above as the fifth case [24]). That is to say, our case report caused by both $P$. canis and $P$. dagmatis was the second to be documented, to the best of our knowledge.

The three isolates of Gram-negative coccobacilli with positive catalase and oxidase reactions were finally identified as $P$. dagmatis for strain-A, P. canis for strain-B, and $P$. canis for strain-C, respectively. ID-Test HN20 panels correctly identified strain-A as $100 \% P$. dagmatis, but misidentified both the strain-B and strain-C as $100 \%$ P. multocida. On the contrary, Vitek 2 GN cards (Nippon sysmex bioMérieux) misidentified the strain-A as P. pneumotropica, but correctly identified both the strain-B and strain- $\mathrm{C}$ as $P$. canis.

The species name $P$. pneumotropica in the Vitek 2 system database (Nippon sysmex bioMérieux) might be $P$. dagmatis, formerly known as $P$. pneumotropica type Henricksen. It is with this fact that the Vitek 2 system database (Nippon sysmex bioMérieux) contained only $P$. multocida, $P$. canis, $P$. aerogenes, and $P$. pneumotropica for identification, and $P$. dagmatis was not included in the database until now. Suggestions should be made to manufacturers to improve their database; however, it is also important for routine clinical microbiology laboratories to know the limitation of the commercial identification systems, such as Vitek 2 (Nippon sysmex bioMérieux) and ID-Test HN20 rapid systems (Nissui Pharmaceutical). As not every laboratory has equipped to handle molecular assays, it will certainly help clinical microbiologists to remember that differential biochemical properties of 
Table 4. Differential phenotypic characteristics of Pasteurella dagmatis and Pasteurella pneumotropica.

\begin{tabular}{lcc}
\hline & Pasteurella dagmatis & Pasteurella pneumotropica \\
\hline Catalase & + & + \\
Oxidase & + & + \\
Ornithine decarboxylase & - & + \\
Indole production & + & + \\
Urease activity & + & + \\
ONPG\# reaction & - & + \\
\hline
\end{tabular}

\#: ortho-nitrophenyl- $\beta$-D-galactopyranoside

oxidase, catalase, ornithine decarboxylase, urease activity, and indole production as shown in Table 4, fermentation of maltose, mannose, sucrose, and glucose are given by ID-Test HN20 panels (Nissui Pharmaceutical) or Vitek 2 GN cards (Nippon sysmex bioMérieux).

In addition, although ID-Test HN20 Rapid panels (Nissui Pharmaceutical) actually contained $P$. canis in the database, strain-B and strain- $\mathrm{C}$ were both misidentified as $P$. multocida. These findings apparently indicated that widely used identification systems prove to be unreliable in the accurate identification of Pasteurella species, especially $P$. dagmatis. Therefore, the remarkably underestimated frequency of isolation of $P$. dagmatis may possibly be ascribed to the misidentification of the species.

Conflicts of interest: The authors have declared that no conflict of interest exists.

\section{REFERENCES.}

1. Smith JE. Studies on Pasteurella septica. II. Some cultural and biochemical properties of strains from different host species. J Comp Pathol Ther. 1958; 68: 315.

2. Owen CR, Buker ED, Bell JE. Pasteurella multocida in animals' mouths. Rocky Mt Med J. 1968; 65: 45-46.

3. Bailie WE, Stowe EC, Schmitt AM. Aerobic bacterial flora and nasal fluids of canines with reference to bacteria associated with bites. J Clin Microbiol. 1978; 7: 223231.

4. Ashley BD, Noone M, Dwarakanath AD, Malnick H. Fatal Pasteurella dagmatis peritonitis and septicaemia in a patient with cirrhosis: a case report and review of the literature. J Clin Pathol. 2004; 57: 210-212.

5. Hubbert WT, Rosen MN. Pasteurella multocida infection due to animal bite. Am J Public Health. 1970; 60: 1103-1108.

6. Weber DJ, Wolfson JS, Swartz MN, Hooper MN. Pasteurella multocida infections: report of 34 cases and review of the literature. Medicine (Baltimore). 1984; 63: 133-154.

7. Holst E, Rollof J, Larsson L, Nielsen JP. Characterization and distribution of Pasteurella species recovered from infected humans. J Clin Microbiol. 1992; 30: 2984-2987.

8. Hubbert WT, Rosen MN. 1970. Pasteurella multocida infection in man unrelated to animal bite. Am J Public Health. 1970; 60: 1109-1117.

9. Raffi F, Barrier J, Daron D, Drugeon HB, Nicolas F, Courteu AL. Pasteurella multocida bacteremia: report of thirteen cases over twelve years and review of the litera- ture. Scand J Infect Dis. 1987; 19: 385-393.

10. Dendle C, Looke D. Review article: Animal bites: an update for management with a focus on infection. Emerg Med Australas. 2008; 20: 458-467.

11. Heydemann J, Heydemann JS, Antony S. Acute infection of a total knee arthroplasty caused by Pasteurella multoci$d a$ : a case report and a comprehensive review of the literature in the last 10 years. Int J Infect Dis. 2010; 14S: e242-e245.

12. Fajfar-Whetstone CJ, Coleman L, Biggs ER, Fox BC. Pasteurella multocida septicemia and subsequent Pasteurella dagmatis septicemia in a diabetic patient. J Clin Microbiol. 1995; 33: 202-204.

13. Neilan BA, Jacobs D, Del Dot T, Blackall LL, Hawkins P R, Cox PT, Goodman AE. rRNA sequences and evolutionary relationships among toxic and nontoxic cyanobacteria of the genus Microcystis. Int J Syst Bacteriol. 1997; 47: 693-697.

14. Matsumoto T, Kawakami Y, Oana K, Honda T, Yamauchi K, Okimura Y, Shiohara M, Kasuga E. First isolation of Dysgonomonas mossii from intestinal juice of a patient with pancreatic cancer. Arch Med Res. 2006; 37: 914-916.

15. Mosallanejad B, Avizeh R, Ghadiri AR, Moarrabi A, Naddaf H, Jamshidian M. First report of Pasteurella dagmatis isolation from a bitch urine in Iran. Iran J Veter Res. 2008; 25: 384-386.

16. Gump DW, Holden RA. 1972. Endocarditis caused by a new species of Pasteurella. Ann Intern Med. 1972; 76: 275-278.

17. Allison K, Clarridge JE III. Long-term respiratory tract infection with canine-associated Pasteurella dagmatis and Neisseria canis in a patient with chronic bronchiectasis. J Clin Microbiol. 2005; 43: 4272-7274.

18. Guillar T, Duval V, Jobart R, Brasme L, David C, de Champs C, Begin M, Dehoux E. Dog bite wound infection by Pasteurella dagmatis misidentified as Pasteurella pneumotropica by automated system Vitek 2. Diagn Microbiol Infect Dis. 2009; 65: 347-348.

19. Talan DA, Citron DM, Abrahamian FM, Moran GJ, Goldstein EJ. N Engl J Med. 1999; 340: 85-92.

20. Ramdeen GD, Smith RJ, Smith EA, Baddour LM. Pasteurella multocida tonsillitis: case report and review. Clin Infect Dis. 1995; 20: 1055-1057.

21. Rosenbach KA, Poblete J, Larkin I. Prosthetic valve endocarditis caused by Pasteurella dagmatis. South Med J. 2001; 94: 1033-1035.

22. Peel MM. Dog-associated bacterial infections in humans: isolates submitted to an Australian reference laboratory, 1981-1992. Pathology. 1993; 25: 379-384.

23. Dupuy O, Garrabé E, Bordier L, Boyer B, Goasguen O, Mayaudon H, Bauduceau B. Pasteurella dagmatis spondylodiscitis in a diabetic patient. Rev Med Interne. 2006; 27: 803-804. 
24. Zbinden R, Sommerhalder P, von Wartburg U. Co-isolation of Pasteurella dagmatis and Pasteurella multocida from cat-bite wounds. Eur J Clin Microbiol Infect Dis. 1988; 7: 203-204.

Received: April 12, 2011 / Accepted: July 11, 2011
Address for correspondence:

Yoshiyuki Kawakami, Ph.D., Professor

Division of Infection Control and

Microbiological Regulation,

Department of Health and Medical Sciences,

Shinshu University Graduate School of Medicine,

Matsumoto, 390-8621, Japan.

Phone: +81-263-37-2381; Fax: +81-263-37-2370;

E-mail: yk23724@shinshu-u.ac.jp 